\title{
Emotional face processing in adolescents with childhood sexual abuse- related posttraumatic stress disorder, internalizing disorders and healthy controls
}

\author{
Marie-José van Hoof ${ }^{\mathrm{a}, \mathrm{b}, \mathrm{c}, *}$, Bianca G. van den Bulk ${ }^{\mathrm{c}, \mathrm{d}}$, Serge A.R.B. Rombouts, ${ }^{\mathrm{c}, \mathrm{e}}$, \\ Mirjam A.W. Rinne-Albers ${ }^{\mathrm{a}}$, Nic J.A. van der Wee ${ }^{\mathrm{c}, \mathrm{g}}$, Marinus H. van IJzendoorn ${ }^{\mathrm{c}, \mathrm{d}, \mathrm{h}}$, \\ Robert R.J.M. Vermeiren ${ }^{\mathrm{a}, \mathrm{c}, \mathrm{i}}$
}

a Curium-LUMC, Department of Child and Adolescent Psychiatry, Leiden University Medical Center, Leiden, The Netherlands

b YOEP (Yulius Onderwijs zorg Expertise Partners), Warmond, The Netherlands

${ }^{c}$ Leiden Institute for Brain and Cognition (LIBC), Leiden University, Leiden, The Netherlands

d Department of Child and Family Studies, Faculty of Social Sciences, Leiden University, The Netherlands

e Institute of Psychology, Leiden University, The Netherlands

f Department of Radiology, Leiden University Medical Center, Leiden, The Netherlands

${ }^{g}$ Department of Psychiatry, Leiden University Medical Center, Leiden, The Netherlands

h School of Pedagogical and Educational Sciences, Erasmus University, Rotterdam, The Netherlands

${ }^{\mathrm{i}}$ VU Medical Center, Department of Child and Adolescent Psychiatry, Amsterdam, The Netherlands

\section{A R T I C L E I N F O}

\section{Keywords:}

fMRI

Adolescents

CSA-related PTSD

Childhood sexual abuse

Posttraumatic stress disorder

Internalizing disorders

Anxiety disorder

Depressive disorder

Emotional face processing

Attentional bias

\section{Introduction}

About one in ten children worldwide experience Childhood Sexual Abuse (CSA; Stoltenborgh et al., 2011), with all too often long lasting and devastating consequences. These include a variety of psychiatric disorders related to emotion dysregulation, that usually last into adulthood (Anda et al., 2006; Fergusson et al., 2013). In order to develop early interventions and personalize treatment to reduce the impact of CSA, better insight in the neural sequelae of CSA, especially in adolescence, is warranted. While there are several behavioral and neurobiological studies on sequelae of CSA (Fergusson et al., 2013), only few studies (e.g. Garrett et al., 2012) have looked into underlying brain mechanisms. Since emotional abuse and neglect are common findings in CSA and psychiatric disorders and emotion regulation is all too often disturbed in adolescents with CSA, as well as in CSA-related psychiatric disorders like posttraumatic stress disorder (PTSD) and anxiety and depressive disorders, it is of interest to focus on this concept. Disturbed emotion regulation can be observed through negative attention bias, i.e. the tendency to direct attention to threats or negative emotions expressed by others (Bar-Haim et al., 2007; Masten et al., 2008; Pine et al., 2005; Romens and Pollak, 2012).

Until recently, attention bias in CSA-related PTSD has been predominantly studied in observational studies, focusing for example on emotional reactivity, i.e. reaction time in relation to viewing emotional faces. In these experiments, it was shown that emotional face processing is distinct in maltreated children, some of whom experienced CSA or depression, as compared to non-abused children and adolescents (e.g. Masten et al., 2008; Pine et al., 2005; Romens and Pollak, 2012).

\footnotetext{
* Corresponding author at: Curium-LUMC, Department of Child and Adolescent Psychiatry, Leiden University Medical Center, P.O. Box 15, 2300 AA Leiden, The Netherlands.

E-mail address: M.J.van_Hoof@lumc.nl (M.-J. van Hoof).
} 
However, results were inconsistent: some studies reported attention bias away from threat, finding slower reaction times in the maltreated group (Pine et al., 2005), other studies reported attention bias towards threat, finding faster reaction times in the maltreated group (Masten et al., 2008). In addition to behavioral measurement of attention bias, fMRI allows study of neural mechanisms involved in the processing of emotional information. The question is whether CSA-related PTSD diagnosis compared to a diagnosis of depression or anxiety disorder is specifically associated with attentional bias and emotional face processing, as measured through emotional reactivity towards emotional faces and by identification of neural correlates. We therefore studied attentional bias and emotional face processing in adolescents with CSArelated PTSD and in adolescents with internalizing disorders and healthy control adolescents with functional MRI (fMRI), using a nonpassive emotional faces task (Van den Bulk et al., 2013).

Emotional face processing fMRI studies in maltreatment-related PTSD in adolescents are relatively scarce (Cisler et al., 2013; Crozier et al., 2014; Fusar-Poli et al., 2009; Lenow et al., 2014). Some of the larger studies on adolescents with sexual assault have partially overlapping inclusion criteria with the CSA-related PTSD sample of this study (Cisler et al., 2015; Garrett et al., 2012; Wolf and Herringa, 2016). These studies showed hyper-activation of either amygdala, hippocampus, insula, dACC and/or PFC and/or hypo-activation of the dIPFC in the PTSD group compared to controls when viewing fearful or neutral faces. Assuming that traumatic stress influences the development of brain regions important for emotion processing, it is suggested (Garrett et al., 2012) that previous trauma or current PTSD symptoms prompted these brain regions to hyperactivate to trauma-related stimuli. An alternative explanation proposed was that adolescents with PTSD are hypersensitive to threat, even before having experienced trauma (Garrett et al., 2012).

In adolescents with depression and/or anxiety disorders, MRI studies on attentional bias and emotional face processing demonstrated attentional bias to threat and fearful faces, but not to neutral faces (e.g. Hall et al., 2014; Hommer et al., 2014; Krain Roy et al., 2008; Waters et al., 2014). Trait anxiety was positively associated with attention bias for angry faces (Telzer et al., 2008). The common denominator for emotional face processing in depression and/or anxiety disorders was hyperactivation of the amygdala in relation to fearful faces (Hall et al., 2014; Swartz et al., 2014a, 2014b), deactivation of the rACC (Hall et al., 2014; Price et al., 2014; Swartz et al., 2014a), insula (Hall et al., 2014; Henje Blom et al., 2015) and superior temporal gyrus (STG; Hall et al., 2014). In order to determine whether diagnostic group is associated with attentional bias and emotional face processing, we directly compare a CSA-related PTSD and an internalizing disorders group on the same fMRI task, simultaneously measuring dimensional psychiatric symptoms.

The aim of this study is to investigate neural correlates of emotional face processing in adolescents with CSA-related PTSD versus adolescents with internalizing disorders and healthy controls. We focused on symptoms of posttraumatic stress, dissociation and depression, given the overlap in symptomatology between both clinical groups. We expected discrepancies in attention bias and emotional face processing between the included groups based on differential degrees of emotional maltreatment or neglect and experienced threat, leading to differential reactions to threat, when measuring reactivity to emotional faces and identifying neural correlates. In line with the literature (Bar-Haim et al., 2007; Cisler et al., 2013, 2015; Crozier et al., 2014; Garrett et al., 2012; Lenow et al., 2014; Masten et al., 2008; Pine et al., 2005; Romens and Pollak, 2012; Wolf and Herringa, 2016) we had three hypotheses. Firstly, we hypothesized adolescents with CSA-related PTSD to have a negative attention bias, showing in slower reaction times in response to fearful and neutral faces compared to adolescents with internalizing disorders (only fearful faces) and healthy controls (Garrett et al., 2012). We based this hypothesis on the expectation that CSA-related PTSD lead to difficulty to disengage from threat (Cisler and Koster, 2010), as induced by fearful faces as well as neutral faces, which are interpreted as ambiguous and therefore threatening (Masten et al., 2008; Pine et al., 2005). Secondly, we hypothesized the adolescents with CSA-related PTSD to show more activation in the thalamus and limbic brain areas (like the amygdala, hippocampus, mid-cingulate, and insula) and less activation in the prefrontal brain areas (like the dlPFC) when interpreting emotional faces compared to adolescents with internalizing disorders (less activation in the ACC and (para)hippocampal regions; Price et al., 2014; Swartz et al., 2014a) and healthy controls (Garrett et al., 2012; Hall et al., 2014). Thirdly, we hypothesized that severity of self-reported posttraumatic stress and dissociation symptoms in adolescents with CSA-related PTSD or internalizing disorders would correlate with increased activation of amygdala and decreased activation of dlPFC (Brown and Morey, 2012; Garrett et al., 2012; Hall et al., 2014; Hart and Rubia, 2012; McClure et al., 2007; Patel et al., 2012; Shin and Liberzon, 2010).

\section{Methods}

\subsection{Participants}

From the original sample of 83 participants, 12 participants (CSA $\mathrm{N}=3$, DEP $\mathrm{N}=4, \mathrm{CNTR} N=5$ ) were excluded from the analyses due to: technical problems during scanning (DEP N $=3$, CNTR $N=1$ ), excessive head movement ( $>3 \mathrm{~mm}, \mathrm{CSA} \mathrm{N}=3, \mathrm{CNTR} \mathrm{N}=2$ ), unforeseen clinical features (CNTR N $=1$ ) or anomalous findings reported by the radiologist (DEP $N=1$, CNTR $N=1$ ). This resulted in a final sample of 19 CSA adolescents (all had CSA-related PTSD), 26 adolescents with internalizing disorders (DEP) and 26 healthy controls (CNTR) from the EPISCA study (Emotional Pathways' Imaging Study in Clinical Adolescents) (Van den Bulk et al., 2013). Attachment and clinical characteristics of the three groups were reported separately (Van Hoof et al., 2015).

All participants met the following inclusion criteria: aged 12-20 years, estimated full scale IQ $\geq 80$ as measured by Dutch versions of the Wechsler Intelligence Scales for Children (WISC-III-NL; Wechsler, 1991) or Adults (WAIS-III; Wechsler, 1997), being right-handed, normal or corrected-to-normal vision, sufficient understanding of the Dutch language, no history of neurological impairments and no contraindications for MRI testing (e.g. braces, metal implants, lead tattoos, irremovable piercings, claustrophobia or possible pregnancy). The CSA group was recruited in two psychotrauma centers of child and adolescent psychiatric institutes, using the third National Incidence Study (NIS-3) operationalized definitions of CSA Specific Form of Maltreatment (NIS-3 code; Sedlak, 2001; see Appendix A in Stoltenborgh et al. (2011)). In order to be sure to get a homogeneous group of participants with substantial experiences and to avoid the discussion about whether sexual assault should be considered childhood sexual abuse, an additional inclusion criterion for the CSA group was having experienced sexual abuse during their lifetime more than once by one or more perpetrators in- or outside the family. Additional inclusion criteria for the internalizing disorders group were: being referred for outpatient treatment, a clinical diagnosis of DSM-IV depressive and/or anxiety disorders and no history of CSA. Exclusion criteria for both clinical groups were: (1) a primary DSM-IV diagnosis of Attention Deficit and Hyperactivity Disorder, Oppositional Defiant Disorder, Conduct disorder, Pervasive Developmental Disorders, Tourette's syndrome, Obsessive-Compulsive Disorder, Bipolar Disorder, and Psychotic Disorders, (2) current use of psychotropic medication other than stable use of SSRI's, or amphetamine medication on the day of the scanning, and (3) current substance abuse. The healthy control adolescents were recruited through local advertisement, with the following additional inclusion criteria: no clinical scores, meaning scores below cut-off points for clinical presentation of symptoms, on validated mood and behavioral questionnaires or past or current Anxiety Disorders Interview Schedule (ADIS C/P) DSM-IV classification, no history of traumatic experiences on ADIS C/P and Adult 
Attachment Interview and no current psychotherapeutic intervention of any kind.

Written informed assent and consent was obtained from all adolescents and their parents. Participants received a financial compensation including travel expenses. The medical ethics committee of the Leiden University Medical Centre approved the study.

\subsection{Clinical assessment}

In addition to standard child psychiatric assessment by a child and adolescent psychiatrist, the child and parent versions of the ADIS-C/P (Lyneham et al., 2007; Silverman et al., 2001) were used to obtain a DSM-IV-based classification of anxiety and depressive disorders including PTSD, which determined diagnostic group. The participants with a secondary classification of non-CSA-related PTSD on the ADIS besides a primary diagnosis of an internalizing disorder, were included in the internalizing disorders group. Estimated full scale IQ was acquired with six subtests of the WISC-III-NL (Wechsler, 1991) and the WAIS-III (Wechsler, 1997). After inclusion, three self-reports were used to assess the severity of posttraumatic stress, dissociation and depression; i.e. the Trauma Symptoms Checklist for Children (TSCC; Briere, 1996), the Adolescent Dissociative Experiences Scale (A-DES; Armstrong et al., 1997; Smith and Carlson, 1996) and the Children's Depression Inventory (CDI; Kovačs, 1992; Timbremont et al., 2004). Total scores of the TSCC and CDI and a $\log ^{10}$ transformation of the total score of the ADES, performed to lift the positive skew, were subsequently used in the analyses.

\subsection{Sample characteristics}

See Table 1.

\subsection{Task paradigm}

All adolescents performed an adapted version of the face processing task originally developed by McClure et al. (2007) and Monk et al. $(2003,2006)$ and has been described in detail previously (Van den Bulk et al., 2013, 2014). In short, the task consisted of three constrained state conditions (state questions: 'how afraid are you?', 'how happy are you?' and 'how wide is the nose?') and one unconstrained (passive viewing) state condition. After state presentation, participants viewed 21 emotional faces ( 7 fearful, 7 neutral and 7 happy facial expressions) per attention state, which they had to rate on a four-point rating scale referring to the presented state (1. not at all, 2. a little, 3. quite and 4. very). During the task, reaction times and subjective scoring of the different emotional faces (fearful, happy or neutral) were recorded for behavioral analyses.

All trials had the same structure: first participants were presented with one of the state questions for 4000 milliseconds which was followed by a centrally located fixation cross with a jittered interval between 500 and 6000 milliseconds. Thereafter, one of the pictures was shown for 3000 milliseconds again followed by a centrally located fixation cross (Fig. 1). When the participant did not respond within 3000 milliseconds the task proceeded to the next trial. The trials during which participants did not respond were recorded as missing trials ( $1,88 \%$ of all trials across all participants), and were not included in the behavioral and fMRI analyses. There were 21 trials per condition (one of the state questions $\times$ one of the emotions) and 252 trials in total. We used a mixed design in which trials were event related within blocks (state questions). We are aware of the ongoing debate whether "neutral" faces exist, or whether the term "ambiguous" faces should be used (e.g. Tahmasebi et al., 2012), but for consistency with our previous paper we use the term 'neutral' faces.

\section{5. fMRI data acquisition}

Data were acquired using a 3.0 T Philips Achieva (Philips, Best, The Netherlands) scanner at the Leiden University Medical Centre. Stimuli were presented onto a screen located at the head of the scanner bore and viewed by participants by means of a mirror mounted to the head coil assembly. First, a localizer was obtained for each participant. Subsequently, T2*-weighted Echo-Planar Images (EPI) $(\mathrm{TR}=2200 \mathrm{~ms}$, $\mathrm{TE}=30 \mathrm{~ms}$, flip angle $=80^{\circ}, 80 \times 80$ matrix, FOV $=220,38$ slices of thickness $2.72 \mathrm{~mm}$ ) were obtained during three functional runs of 192 volumes each. Each run had two additional volumes, which were discarded to allow for equilibration of T1 saturation effects. Also, a sagittal 3-dimensional gradient-echo T1-weighted image was acquired for registration purposes with the following scan parameters: $\mathrm{TR}=9.8 \mathrm{~ms}$; TE $=4.6 \mathrm{~ms}$; flip angle $8^{\circ} ; 140$ sagittal slices; no slice gap; FOV $=192 \times 152$ matrix; FOV $=224 \times 177 \times 168 \mathrm{~mm}, 140$ sagittal slices; no slice gap; $1.16 \times 1.16 \times 1.20 \mathrm{~mm}$ voxels.

We used a mock-scanner to familiarize all participants with the MRI scanner. Participants were placed in the mock-scanner and they received information about the scanning procedure. In addition, we

Table 1

Sample characteristics and psychiatric symptom scores.

\begin{tabular}{|c|c|c|c|c|c|c|c|c|c|c|}
\hline Sample characteristics & \multicolumn{2}{|c|}{$\operatorname{CSA}(n=19)$} & \multicolumn{2}{|c|}{ DEP $(n=26)$} & \multicolumn{2}{|c|}{ CNTR $(n=26)$} & \multirow{2}{*}{$\frac{\chi^{2}}{0.28}$} & \multirow{2}{*}{$\frac{d f}{2}$} & \multicolumn{2}{|l|}{$p$} \\
\hline Females/Males & $17 / 2$ & & $22 / 4$ & & $23 / 3$ & & & & 0.01 & \\
\hline Number of stable SSRI use & 2 & & 1 & & - & & & & & \\
\hline \multirow[t]{2}{*}{ Number meeting PTSD criteria } & $19^{\mathrm{a}}$ & & - & & - & & & & & \\
\hline & Mean & SD & Mean & SD & Mean & SD & $\eta^{2}$ & $F_{(2,70)}$ & $p$ & Significant group differences \\
\hline Age in years & 16.62 & 1.78 & 15.98 & 1.45 & 15.25 & 1.64 & 0.11 & 4.03 & 0.02 & $\mathrm{CSA}>\mathrm{DEP}>\mathrm{CNTR}$ \\
\hline Full scale IQ & 99.89 & 9.10 & 105.12 & 8.66 & 106.58 & 7.77 & 0.10 & 3.60 & 0.03 & CSA $<$ DEP $<$ CNTR \\
\hline Psychiatric symptom scores & Mean $^{\text {b }}$ & SD & $\operatorname{Mean}^{\mathrm{c}}$ & SD & Mean & SD & $\eta^{2}$ & $F_{(2,68)}$ & $p$ & Significant group differences \\
\hline TSCC score & 45.74 & 22.92 & 41.90 & 22.85 & 16.26 & 12.50 & 0.31 & 13.23 & 0.00 & CSA, DEP $>$ CNTR \\
\hline A-DES score & 1.65 & 0.42 & 1.46 & 0.44 & 1.20 & 0.46 & 0.18 & 6.37 & 0.03 & $\mathrm{CSA}^{*}, \mathrm{DEP}^{* *}>\mathrm{CNTR}$ \\
\hline CDI score & 15.15 & 6.89 & 17.75 & 8.89 & 4.17 & 3.36 & 0.49 & 27.85 & 0.00 & CSA, DEP $>$ CNTR $^{*}$ \\
\hline
\end{tabular}

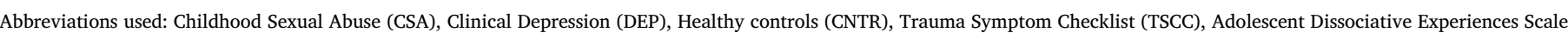

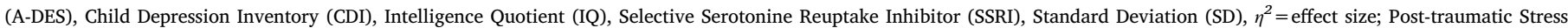
Disorder (PTSD), MANOVA analyses used both demeaned age in years and demeaned IQ.

a According to ADIS 18 met full PTSD criteria and one failed the interference criterium only slightly.

$\mathrm{b} \mathrm{n}=18$.

c $\mathrm{n}=24$.

* Significant at $p<0.05$

$* *$ Significant at $p<0.01$. 


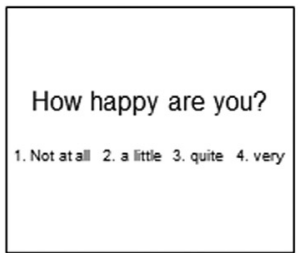

$4000 \mathrm{~ms}$.

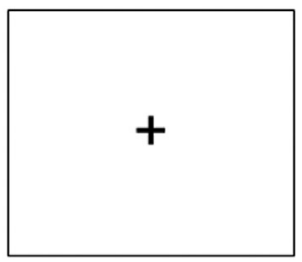

Jittered

$500-6000 \mathrm{~ms}$.

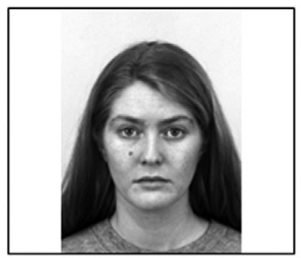

$3000 \mathrm{~ms}$.

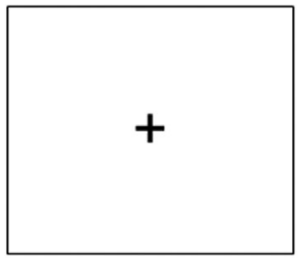

Jittered

$500-6000 \mathrm{~ms}$

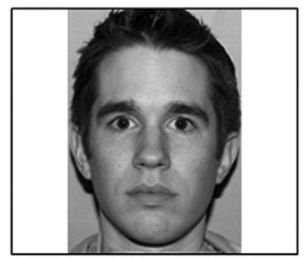

$3000 \mathrm{~ms}$.

Time

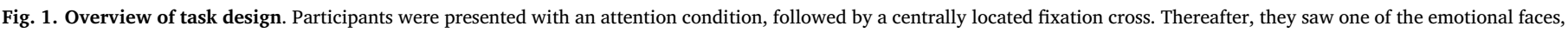

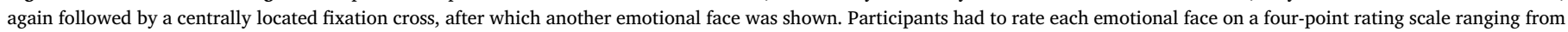
"not at all" to "very," based on the presented attention condition (5).

presented the scanner sounds on a laptop so that participants knew what to expect while in the MRI scanner.

\subsection{Imaging data analysis}

We used SPM8 (Wellcome Department of Cognitive Neurology, London) to analyze the acquired data. Data was preprocessed using the following steps: 1. realignment of functional time series to compensate for small head movements and differences in slice timing acquisition, 2. registration and normalization of functional volumes (from EPI to individual structural $\mathrm{T} 1$ and thereafter to the $\mathrm{T} 1$ template), 3. spatially smoothing the functional volumes with an $8 \mathrm{~mm}$, full-width at halfmaximum isotropic Gaussian kernel. The normalization algorithm used a 12-parameter affine transformation together with a nonlinear transformation involving cosine basis functions and resampled the volumes to three mm cubic voxels. The MNI (Montreal Neurological Institute) 305 stereotaxic space templates (Cocosco et al., 1997) were used for visualization and all results are reported in this template, which is an approximation of Talairach space (Talairach and Tournoux, 1988).

Individual subjects' data were analyzed using the general linear model in SPM8. The fMRI time series were modeled by a series of events convolved with a canonical hemodynamic response function (HRF). The state questions were modeled separately as 4000 millisecond events and were added as covariates of no interest. The picture presentation of each emotional face was modeled as a 3000 millisecond duration event. In the model, the picture presentation was further divided in twelve separate function trials (four state questions by three expressed emotions). The modeled events were used as regressors in a general linear model along with a basis set of cosine functions that highpass filtered the data. The least squares parameter estimates of the height of the best-fitting canonical HRF for each condition were used in pair wise contrasts (e.g. all faces vs. fixation). The resulting contrast images, computed on a subject-by-subject basis, were submitted to group analyses. At the group level, contrasts between conditions were computed by performing full factorial models on these images, treating subjects as a random effect. Task-related responses were considered significant if they consisted of at least 10 contiguous voxels at a corrected threshold of $p<0.05$ (FDR corrected).

We used the MarsBaR toolbox for use with SPM8 (http://marsbar. sourceforge.net/; 44) to perform region of interest (ROI) analyses to further investigate patterns of activation. Based on the current literature about CSA, PTSD, major depression and anxiety disorders we used a priori and anatomically defined ROIs: (all left and right) dorsolateral PFC (DLPFC), amygdala, insula, thalamus, mid cingulum, hippocampus. The templates for the anatomically defined ROIs were derived from the MarsBaR toolbox (AAL-templates).

\subsection{Data analyses}

For the behavioral and fMRI analyses we used the same analysis frame work presented before (Van den Bulk et al., 2013, 2014). We compared groups regarding total scores on the TSCC, A-DES and CDI using MANCOVA (with simple bootstrapping). To analyze the effects of emotional faces on subjective scoring, we performed separate repeated measure ANCOVA's for each state question (three in total). We used a group ( 3 levels) $\times$ emotion ( 3 levels) design per state question. The three state questions were analyzed separately because values of the scores represent different interpretations for each state. For reaction time, a 3 (groups) $\times 3$ (state questions) $\times 3$ (emotions) repeated measure ANCOVA was performed. Because of the significant differences in age and IQ, we included demeaned age and IQ as covariates in all analyses. For all analyses, Greenhouse-Geisser correction (GG-corr.) was applied in case sphericity was not assumed and we used Bonferroni correction for post-hoc comparisons. Behavioral data were checked for outliers (by using boxplots and z-scores), but no consistent outliers were detected (z-score $>3.29$ ).

The results of the ROI analyses were submitted to repeated measure ANCOVA's in SPSS. For each set of regions, we used a 3 (group) $\times 2$ (hemisphere) $\times 4$ (state question) $\times 3$ (emotion) repeated measure ANCOVA. We included hemisphere as a factor in the repeated measure ANCOVA's to limit the number of statistical analyses. We included demeaned age and estimated IQ level as covariates. Again, GreenhouseGeisser correction (GG-corr.) was applied in case sphericity was not assumed and post hoc comparisons within a repeated measure ANCOVA were Bonferroni corrected for multiple comparisons. ROI data were also checked for outliers, but no consistent outliers were detected.

\section{Results}

\subsection{Behavioral data}

\subsubsection{Co-morbidity}

Of the participants with CSA-related PTSD in this sample 11/19 (57.1\%) had a comorbid anxiety disorder, $7 / 19$ (38.1\%) had a comorbid depressive disorder and 7/19 (38.1\%) had both comorbid anxiety and depressive disorders according to the ADIS. Of the participants in the internalizing disorders group in this sample, $8 / 26$ (30.8\%) individuals had PTSD comorbidity according to the ADIS. In the internalizing disorders group 5/26 (20\%) had only anxiety disorder, $1 / 26(4 \%)$ individuals only had a depressive disorder and 13/26 (50\%) individuals had a combination of anxiety and depressive disorders.

\subsubsection{Self-reported symptomatology}

A significant effect for clinical group (partial $\eta^{2}=0.28, F_{(6}$, $\left.{ }_{116)}=7.33, p<0.001\right)$ was found for trauma (TSCC) $\left(F_{(2,68)}=13.23\right.$, $p<0.001$, partial $\eta^{2}=0.31$ ), dissociation (A-DES) $\left(F_{(2,68)}=6.37\right.$, $p=0.03$, partial $\eta^{2}=0.18$ ), and depression scores (CDI) $\left(F_{(2,68)}=27.85, p<0.001\right.$, partial $\left.\eta^{2}=0.49\right)$. Post-hoc comparisons showed significant higher levels of self-reported symptomatology in the CSA and DEP groups than in the CNTR group (TSCC and CDI both at $p<0.001$; A-DES CNTR-DEP group at $p=0.04$ and CNTR-CSA group at $p=0.004$ ), but demonstrated no significant differences in self-reported 
symptomatology between both clinical groups (see Table 1).

\subsubsection{Subjective rating of emotional faces}

The ANCOVA for the state induced by the question 'how wide is the nose?' resulted in a main effect of emotion $\left(F_{(2,132)}=393.54, p<0.001\right.$, partial $\eta^{2}=0.86$ ), in which subjective scoring for nose width was higher for happy faces compared to both fearful and neutral faces (both $\left.p^{\prime} s<0.001\right)$. Also, scores were higher for fearful faces than for neutral faces $(p<0.001)$. There were no significant main or interaction effects with group.

The ANCOVA for the state induced by the question 'how afraid are you?' showed a main effect for group $\left(F_{(2,66)}=5.29, p<0.01\right.$, partial $\left.\eta^{2}=0.14\right)$, a main effect for emotion $\left(F_{(2,132)}=57.72, p<0.001\right.$, partial $\eta^{2}=0.47$, GG-corrected), and a group $\times$ emotion interaction $\left(F_{(4,132)}=3.88, p<0.01\right.$, partial $\eta^{2}=0.11$, GG-corrected). Overall, CSA and DEP adolescents reported being more afraid of fearful faces than CNTR adolescents $(p<0.01$ and $p<0.05$ respectively). In addition, CSA adolescents reported being more afraid of neutral faces compared to CNTR adolescents $(p<0.05)$, and the DEP group (marginally significant, $p=0.06$ ). There were no significant group differences for happy faces (all $p$ 's $>0.42$ ).

The ANCOVA for the state induced by the question 'how happy are you?' resulted in a main effect of group $\left(F_{(2,66)}=4.72, p<0.05\right.$, partial $\left.\eta^{2}=0.13\right)$, a main effect of emotion $\left(F_{(2,132)}=121.89, p<0.001\right.$, partial $\eta^{2}=0.65$, GG-corrected) and an emotion $\times$ group interaction effect $\left(F_{(4,132)}=2.82, p<0.05\right.$, GG-corrected, partial $\left.\eta^{2}=0.08\right)$. Overall, CSA and DEP adolescents reported being less happy when viewing fearful faces than healthy control group adolescents $(p<0.05$ and $p<0.005$ respectively).

\subsubsection{Reaction times}

The ANCOVA resulted in a main effect for group $\left(F_{(2,66)}=4.75\right.$, $p<0.05$, partial $\left.\eta^{2}=0.13\right)$, a main effect of emotion $\left(F_{(2,132)}=5.47\right.$, $p<0.01$, partial $\eta^{2}=0.08$ ) and an interaction effect of state $\times$ emotion $\left(F_{(4,264)}=10.17, p<0.001\right.$, partial $\left.\eta^{2}=0.13\right)$. Reaction times were higher for the CSA group than for the DEP group $(p<0.01)$, but not for the CNTR group $(p<0.20)$. Also there was no significant difference between the DEP and CNTR group $(p<0.50)$. Overall, participants reacted slower to fearful and neutral faces than to happy faces (both $p$ ' $<0.05)$. There was no significant difference between fearful and neutral faces $(p<0.50)$ Furthermore, reaction times within the state 'how afraid are you?', were higher for fearful and neutral faces than for happy faces (both $p$ 's $<0.001$ ), while there was no difference between fearful and neutral faces $(p<0.50)$ (Fig. 2).

\subsection{Whole brain analyses}

To examine whether the task activated the expected brain regions, we performed whole brain analyses for the complete sample $(\mathrm{N}=71)$. As can be seen in Fig. 3, the analyses of all faces $>$ fixation resulted in robust activation in brain regions related to emotional faces processing, including the bilateral amygdala, bilateral insula, bilateral thalamus and bilateral PFC. The whole brain contrasts for each emotion (fearful faces $>$ fixation, happy faces $>$ fixation, neutral faces $>$ fixation) and the contrasts to test the differential effects of emotion processing (fearful face $>$ neutral faces, happy faces $>$ neutral faces, fearful faces $>$ happy faces and happy faces $>$ fearful faces) across all participants are presented in Supplemental Table 1 and Supplemental Figure 1.

Because we were primarily interested in group differences, we performed direct comparisons between the three groups on whole brain level for all previously mentioned contrasts with the use of full factorial model ANOVA's. These analyses did not result in significant differential activation between the three groups.

\subsection{Region of Interest analyses}

We performed 3 (groups) $\times 2$ (hemispheres) $\times 4$ (state question) $\times 3$ (emotion) repeated measure analyses for each set of ROIs (DLPFC, amygdala, insula, thalamus, mid cingulum and hippocampus).

The results showed a significant hemisphere $\times$ state $\times$ emotion $\times$ group interaction effect for the hippocampus $\left(F_{(12,396)}=2.11\right.$, $p<0.05$, partial $\eta^{2}=0.06$ ). Post hoc comparisons showed more right hippocampus activation compared to left hippocampus activation in the CSA group (fearful faces within 'how happy are you?' and fearful, happy and neutral faces 'how wide is the nose?', all $p$ 's $<0.05$ ) and the internalizing disorders group (happy faces within 'how afraid are you?' and neutral faces within 'how wide is the nose?', both $p$ 's $<0.05$ ). Also, for the healthy controls there was more left hippocampus activation for fearful compared to neutral faces within the state question 'how happy are you?' $(p<0.05)$. Finally, for the adolescents with internalizing disorders there was more left hippocampus activation for happy compared to neutral faces within the state question 'how wide is the nose?' $(p=0.005)$.

For mid cingulate cortex, there was a significant hemisphere $\times$ state $\times$ group interaction $\left(F_{(6,198)}=2.27, p<0.05\right.$, partial $\left.\eta^{2}=0.06\right)$. Posthoc comparisons showed more deactivation in left mid cingulate compared to right mid cingulate for the healthy controls ('how afraid are you?' $p<0.05$; 'how wide is the nose?' $p<0.005$ ), the adolescents with CSA-related PTSD ('how afraid are you?' $p<0.01$; 'how wide is the nose?' $p<0.01$ ) and the adolescents with internalizing disorders ('how wide is the nose?' $p<0.05$ ). In addition, there was more right than left deactivation of the mid cingulate for passive viewing within the CSA group. Other regions showed no significant main or interaction effects for group. There were, however, some main and interaction effects for hemisphere, state and emotion (see Appendix).

When excluding the participants with stable SSRI use $(\mathrm{N}=1$ DEP, $\mathrm{N}=2$ CSA), the results of the ROI analyses were comparable and no additional significant main or interaction effects for group were found.

\subsection{Correlation analyses}

To examine whether there is a relation between brain activation and self-report measurements, we performed correlation analyses for the complete sample of $\mathrm{N}=71$ and for all three groups separately in SPSS using demeaned age and IQ as covariates. We included the ROI results for bilateral amygdala and bilateral DLPFC activation and self-reported total scores on the TSCC, A-DES and CDI. There were no significant correlations between any of these variables.

\section{Discussion}

In this article we examined the neural mechanisms of emotional face processing in adolescents with CSA-related PTSD versus internalizing disorders and healthy controls. In accordance with our first hypothesis, the CSA-related PTSD group reacted slower to all emotional faces across all questions than the internalizing disorders group. There was no significant difference in reaction time between the two clinical groups and the healthy controls. Also, all participants reacted slower to fearful and neutral faces compared to happy faces within the state question 'How afraid are you?'. Besides, both clinical groups reported higher levels of subjective fear in response to fearful faces than healthy controls and the CSA-related PTSD group reported higher levels of subjective fear to neutral faces than the internalizing disorders group and the healthy controls. Within the state question 'How happy are you?' the CSA-related PTSD group and the internalizing disorders group reported being less happy when viewing fearful faces than the healthy controls. Contrary to our second and third hypotheses, no significant differences between groups were found on whole brain and on ROI level, and no correlations between levels of self-reported posttraumatic stress, dissociation and depression and brain activation. 

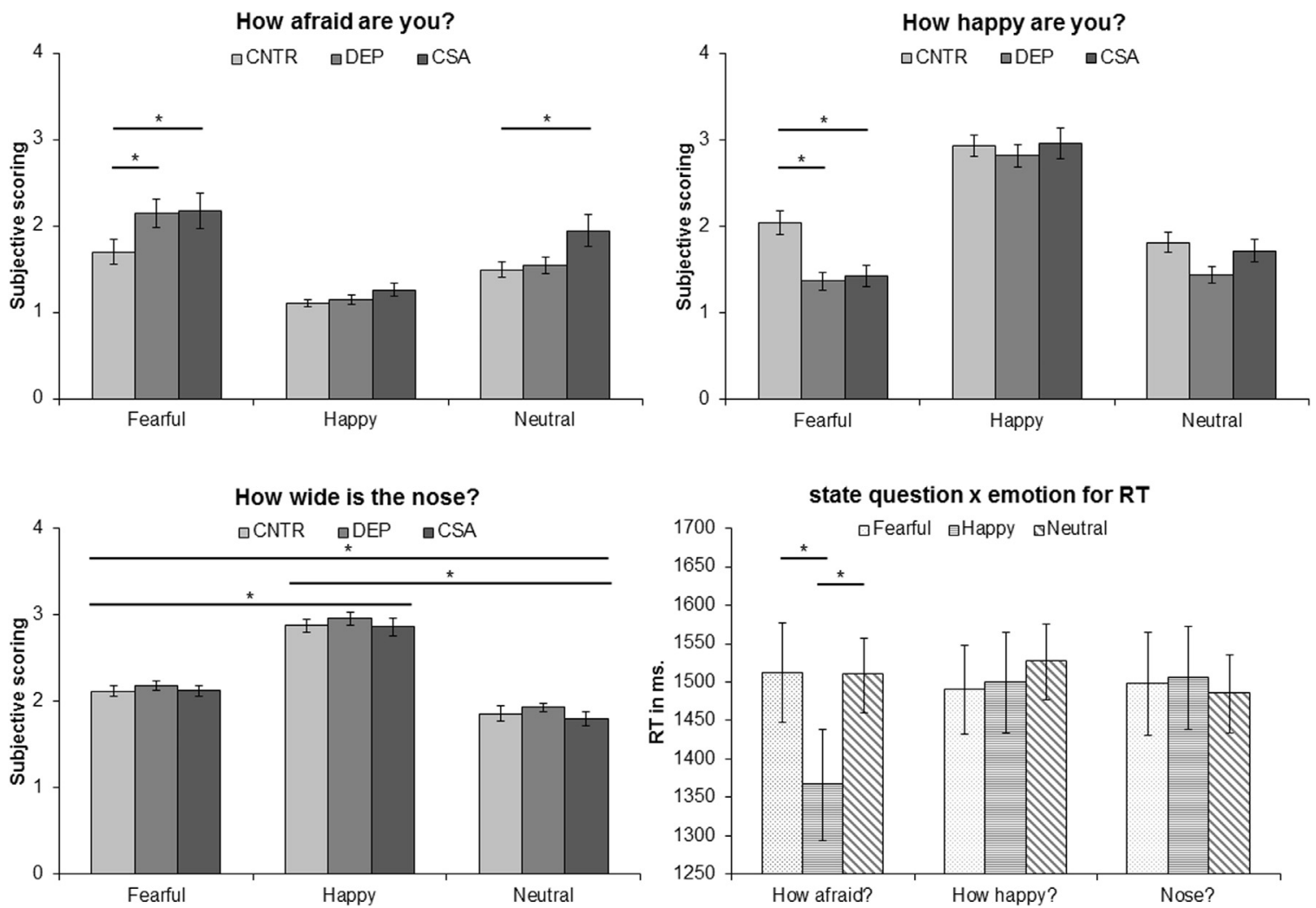

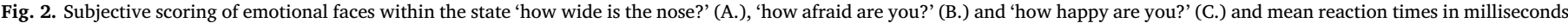
per group (D.) *p $<0.05$.

In line with our first hypothesis we found a negative attention bias in the CSA-related PTSD group: reaction times to emotional faces were slower compared to the internalizing disorders group and the healthy control group. Further, adolescents with CSA-related PTSD experienced higher subjective fear to fearful and neutral faces than healthy controls, while adolescents with internalizing disorders only had higher subjective fear scores compared to healthy controls when viewing fearful faces. These findings suggest that adolescents with CSA-related PTSD are more reactive to neutral emotional faces than adolescents with internalizing disorders and more reactive to fearful and neutral faces

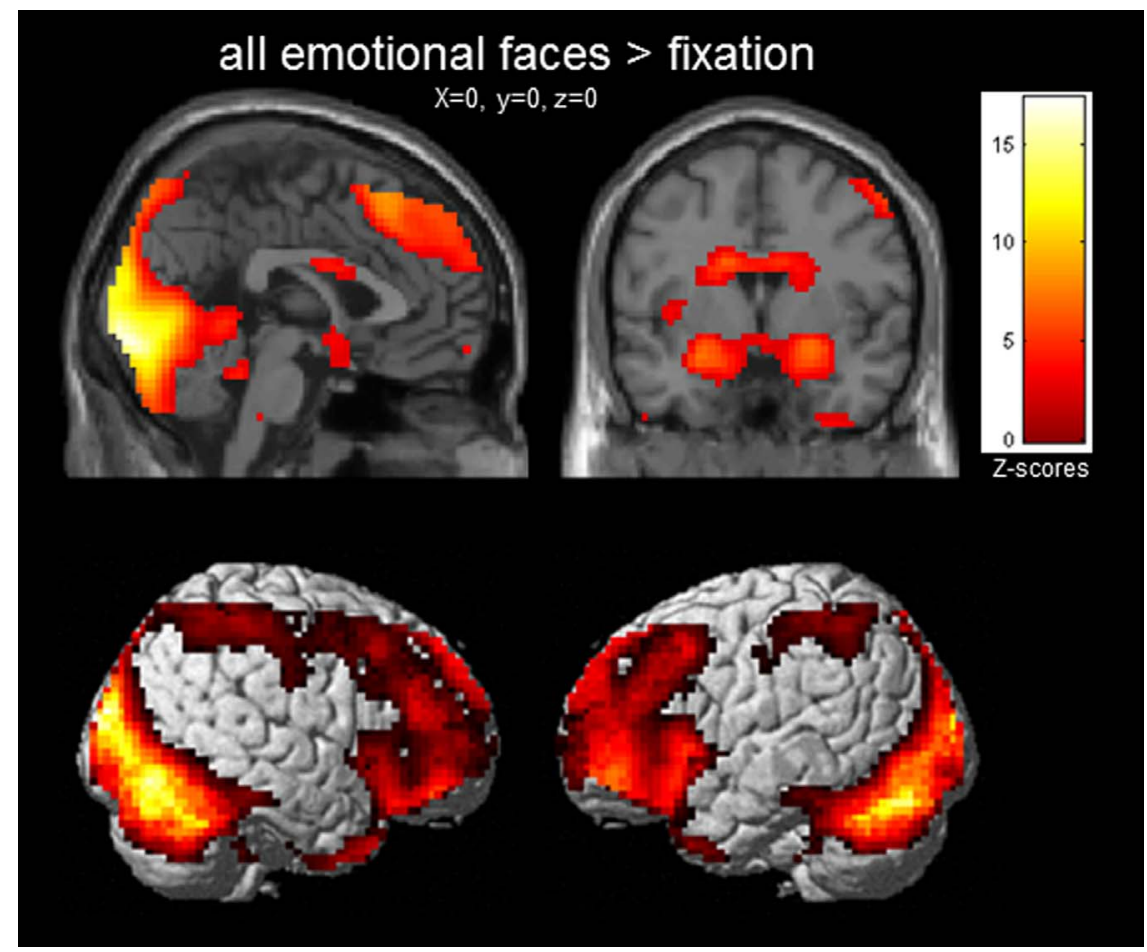

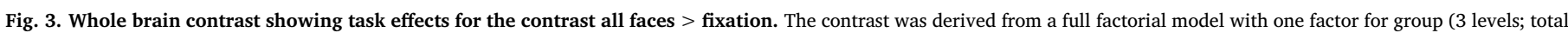
$\mathrm{N}=71$ ) and demeaned age and estimated total IQ as covariates. Thresholding: FDR corrected, $p<0.05$ with at least 10 contiguous voxels. 
compared to healthy controls. However, in line with the literature and the definition we used (Cisler and Koster, 2010; Pine et al., 2005), we interpret this finding as a negative attention bias, due to a need from previous adverse experiences to carefully examine a possible threat even in neutral stimuli. As neutral faces are ambiguous, this may heighten their threat level. Attention bias to the threat will require more time to react and elicit higher subjective fear doing so, as was the case in the CSA-related PTSD group and partly in the internalizing disorders group (for fearful faces only). We assume attention bias is the result of a combination of automatic and strategic emotional face processing in this case, involving heightened threat detection and difficulty to disengage (Cisler and Koster, 2010). Our finding of negative attention bias is consistent with studies in maltreated children and adolescents using a non-morphed emotional faces task like we did, which showed that maltreated youth process threat-related information more slowly than controls (Pine et al., 2005). Other studies (e.g. Masten et al., 2008; Romens and Pollak, 2012) used a heterogeneous sample that may use different attention bias components and strategies, a different paradigm (e.g. visual probe, visual discrimination and identification, or morphed facial emotion identification task), or a different presentation of emotional cues and questions posed, which all may account for inconsistency in attention bias between studies.

Contrary to our second hypothesis, whole-brain analyses nor ROIs revealed group differences. The interaction effects did not survive correction for multiple comparisons. Therefore, we refrain from interpreting these interaction effects as whole-brain or ROI group differences. Nevertheless, the paradigm used in this study has been shown to be valid and functional in measuring emotional face processing in youth (Van den Bulk et al., 2013, 2014; Masten et al., 2008). Though the task was shown to activate adequately, no significant differential amygdala activation was found between groups, contrary to previous findings in passively viewing fear and disgust (Nooner et al., 2014).

Contrary to our third hypothesis and the study by Garrett et al. (2012), no significant relation was found between levels of self-reported posttraumatic stress, dissociation or depression symptoms and ROI activation. Garrett's lab found a significant positive correlation between the severity of PTSD symptoms as measured by the Clinician-Administered PTSD Scale for Children and Adolescents (CAPS-CA; Nader et al., 1996) and activation of the MPFC when viewing fearful faces. Although TSCC (Nooner et al., 2014) and CDI (Pagliaccio et al., 2013) have been associated with brain activation in other studies, these self-reports cannot easily be compared to the CAPS-CA interview (Anderson et al., 1986; Nader et al., 1996). The absence of significant relations between self-reported symptomatology and brain activation might furthermore be explained by the fact that the CSA-related PTSD and internalizing disorders groups appeared to not significantly differ in dimensionally assessed psychiatric symptomatology after they were included based on conventional inclusion criteria for diagnostic group. However, the clinical groups did differ significantly from the healthy control group. It is therefore surprising that no differences between diagnostic groups were found on whole brain or ROI level.

Several factors may explain the absence of group differences on the neural level in the current study. First, group sizes were rather small, given the heterogeneous nature of the conditions under investigation. Power was further decreased because we had to control for two covariates (age and IQ). Second, the CSA-related PTSD group was not compared to a non-CSA-related PTSD group but to an internalizing disorders group, which partly appeared to have PTSD for other reasons than CSA. This group was too small to split. Given the overlap in clinical symptoms, this may have impacted the results. Third, the task may not be sensitive enough to detect group differences on the neural level. The original face attention paradigm developed by Monk, McClure and colleagues (McClure et al., 2007; Monk et al., 2003) was used in children with generalized anxiety disorder and controls. The adapted version of the task we used differed from the original paradigm. Anger as emotion was not displayed and therefore the question 'how hostile is the face?' was left out. This changes the perspective taken by the participant compared to the original task and may account for differences in the possibility to detect group differences of neural correlates. Also, the adaptations made to the original task might have prevented us from finding activation differences between the internal emotion focused state questions (e.g. "how afraid are you?') and the external focused state question ('how wide is the nose?'). Fourth, the task was not tailored to content specificity of the clinical groups. As a recent meta-analysis suggests, greater attention bias toward disorder-congruent relative to disorder-incongruent threat stimuli might make a difference (Pergamin-Hight et al., 2015). Fifth, attention bias variability, i.e. attention fluctuations alternating toward and away from threat, rather than attention bias might have revealed group differences, as recently suggested by Naim et al. (2015). An alternative, but more unlikely explanation may be that internalizing disorders and CSA-related PTSD in adolescents do not substantially differ on the neural level, and that with regard to psychiatric symptomatology they have more in common than usually has been hypothesized (Van Hoof et al., 2015).

\section{Conclusions}

This is the first study that compared CSA-related PTSD adolescents with both adolescents with internalizing disorders and healthy controls, extensively measuring behavioral and neural correlates of emotional face processing at the same time. Our results support the hypothesis of increased negative attention bias towards fearful and neutral faces in adolescents with CSA-related PTSD versus internalizing disorders and healthy controls. It is however remarkable that no neural differences were found between all three groups. Clinical applications of knowledge about attention bias and emotional face processing are interesting future options for diagnostics and treatment of adolescents with CSArelated PTSD or internalizing disorders, e.g. Attention Bias Modification Treatment (Hakamata et al., 2010) or attention control training (Badura-Brack et al., 2015). It may well be that the slower reaction time to fearful and neutral emotional faces in adolescents with CSArelated PTSD interferes with their daily social functioning and prevents them from seeking and accepting help. In that case other strategies are needed first to engage them coming into psychotherapy.

\section{Financial disclosures}

The authors declared no potential conflicts of interests with respect to the authorship and/or publication of this article.

\section{Acknowledgements}

We thank all adolescents and their parents for their participation; the members of the research team (C.I. Gelderblom, P.H.F. Meens) and scientific advisors (M.A. van Buchem, E.A. Crone and specifically N.D.J. van Lang who designed the study) and the Departments of Psychiatry and Radiology of the Leiden University Medical Center (LUMC) for facilitation of this neuroimaging study in multiple respects, e.g. MRI data acquisition and analysis. We also thank all students, mental health professionals and personnel who helped with patient selection and inclusion and data management. M.J. van Hoof was supported by the Psychotrauma center and GGZ Kinderen en Jeugd and a grant from WOP Rivierduinen, B.G. van den Bulk was supported by a grant from the Hersenstichting, M.H. van IJzendoorn was supported by a SPINOZA Prize. S.A. Rombouts was supported by a Vici grant from The Netherlands Organization for Scientific Research (NWO). The study was conducted with a grant from the LUMC.

\section{Appendix A. Supporting information}

Supplementary data associated with this article can be found in the 
online version at http://dx.doi.org/10.1016/j.pscychresns.2017.04. 006.

\section{References}

Anda, R.F., Felitti, V.J., Bremner, J.D., Walker, J.D., Whitfield, C., Perry, B.D., Dube, S.R., Gilles, W.H., 2006. The enduring effects of abuse and related adverse experiences in childhood. A convergence of evidence from neurobiology and epidemiology. Eur. Arch. Psychiatr. Clin. Neurosci. 256 (3), 174-186.

Anderson, J.P., Bush, J.W., Berry, C.C., 1986. Classifying function for health outcome and quality-of-life evaluation. Self-versus interviewer modes. Med. Care 24 (5), 454-469.

Armstrong, J.G., Putnam, F.W., Carlson, E.B., Libero, D.Z., Smith, S.R., 1997. Development and validation of a measure of adolescent dissociation: the Adolescent Dissociative Experiences Scale. J. Nerv. Ment. Dis. 185 (8), 491-497.

Badura-Brack, A.S., Naim, R., Ryan, T.J., Levy, O., Abend, R., Khanna, M.M., McDermott, T.J., Pine, D.S., Bar-Haim, Y., 2015. Effect of attention training on attention bias variability and PTSD symptoms: randomized controlled trials in Israeli and U.S. Combat veterans. AJP Adv. http://dx.doi.org/10.1176/appi.ajp.2015.14121578,

Bar-Haim, Y., Lamy, D., Pergamin, L., Bakermans-Kranenburg, M.J., Van IJzendoorn, M.H., 2007. Threat-related attentional bias in anxious and nonanxious individuals: a meta- analytic study. Am. Psychol. Bull. 133 (1), 1-25.

Briere, J., 1996. Trauma Symptom Checklist for Children (TSCC) Professional Manual. Psychol Assess Resources, Odessa, FL.

Brown, V.M., Morey, R.A., 2012. Neural systems for cognitive and emotional processing in posttraumatic stress disorder. Front. Psychol. 3 (449), 1-14.

Cisler, J.M., Koster, E.H.W., 2010. Mechanisms of attentional biases towards threat in the anxiety disorders: an integrative review. Clin. Psychol. Rev. 30 (2), 203. http://dx. doi.org/10.1016/j.cpr.2009.11.003

Cisler, J.M., Steele, J.S., Smitherman, S., Lenow, J.K., Kilts, C.D., 2013. Neural processing correlates of assaultive violence exposure and PTSD symptoms during implicit threat processing: a network-level analysis among adolescent girls. Psychiatr. Res.: Neuroimaging 214, 238-246.

Cisler, J.M., Sigel, B.A., Kramer, T.L., Smitherman, S., Vanderzee, K., Pemberton, J., Kilts, C.D., 2015. Amygdala response predicts trajectory of symptom reduction during Trauma-Focused Cognitive-Behavioral Therapy among adolescent girls with PTSD. J. Psychiatr. Res. 33-40.

Cocosco, C.A., Kollokian, V., Kwan, R.K.S., Evans, A.C., 1997. BrainWeb: online interface to a 3D MRI simulated brain database. NeuroImage 5, s425.

Crozier, J.C., Wang, L., Huettel, S.A., De Bellis, M.D., 2014. Neural correlates of cognitive and affective processing in maltreated youth with posttraumatic stress symptoms: does gender matter? Dev. Psychopathol. 26 (2), 491-513.

Fergusson, D.M., McLeod, G.F.H., Horwood, L.J., 2013. Childhood sexual abuse and adult development outcomes: findings from a 30-year longitudinal study in New Zealand. Child Abus. Negl. 37, 664-674.

Fusar-Poli, P., Placentino, A., Carletti, F., Landi, P., Allen, P., Surguladze, S., Benedetti, F., Abbamonte, M., Gasparotti, R., Barale, F., Perez, J., McGuire, P., Politi, P., 2009. Functional atlas of emotional faces processing: a voxel-based meta-analysis of 105 functional magnetic resonance imaging studies. J. Psychiatr. Neurosci. 34 (6), $418-432$.

Garrett, A.S., Carrion, V., Kletter, H., Karchemskiy, A., Weems, C.F., Reiss, A., 2012. Brain activation to facial expressions in youth with PTSD symptoms. Depression Anxiety $29,449-459$.

Hakamata, Y., Lissek, S., Bar-Haim, Y., Britton, J.C., Fox, N.A., Leibenluft, E., Ernst, M., Pine, D.S., 2010. Attention Bias Modification Treatment: a meta-analysis toward the establishment of novel treatment for anxiety. Biol. Psychiatr. 68, 982-990.

Hall, L.M.J., Klimes-Dougan, B., Hunt, R.H., Thomas, K.M., Houri, A., Noack, E., Mueller, B.A., Lim, K.O., Cullen, K.R., 2014. An fMRI study in emotional face processing in adolescent major depression. J. Affect. Dis. 168, 44-50.

Hart, H., Rubia, K., 2012. Neuroimaging of child abuse: a critical review. Front. Hum. Neurosci. 6 (3), 1-24.

Henje Blom, E., Connolly, C.G., Ho, T.C., LeWinn, K.Z., Mobayed, N., Han, L., Paulus, M.P., Wu, J., Simmons, A.N., Yang, T.T., 2015. Altered insular activation and increased insular functional connectivity during sad and happy face processing in adolescent major depressive disorders. J. Affect. Dis. 178, 215-223.

Hommer, R.E., Meyer, A., Stoddard, J., Connolly, M.E., Mogr, K., Bradley, B.P., Pine, D.S., Leibenluft, E., Brotman, M.A., 2014. Attention bias to threat faces in severe mood dysregulation. Depression Anxiety 31 (7), 559-565.

Kovačs, M., 1992. Children's Depression Inventory (CDI) Manual. Multi-Health Systems, New York.

Krain Roy, A., Vasa, R.A., Bruck, M., Mogg, K., Bradley, B.P., Sweeney, M., Berman, L, McClure-Tone, E.B., Pine, D.S., CAMS Team, 2008. Attention bias toward threat in pediatric anxiety disorders. J. Am. Acad. Child Adolesc. Psychiatry 47 (10), 1189-1196.

Lenow, J.K., Steele, J.S., Smitherman, S., Kilts, C.D., Cisler, J.M., 2014. Attenuated behavioral and brain responses to trust violations among assaulted adolescent girls. Psychiatr. Res.: Neuroimaging 223, 1-8.

Lyneham, H.J., Abbott, M.J., Rapee, R.M., 2007. Interrater reliability of the Anxiety Disorders Interview Schedule for DSM-IV: child and parent version. J. Am. Acad. Child Adolesc. Psychiatry 46 (6), 731-736.

Masten, C.L., Guyer, A.E., Hodgdon, H.B., McClure, E.B., Charney, D.S., Ernst, M., Kaufman, J., Pine, D.S., Monk, C.S., 2008. Recognition of facial emotions among maltreated children with high rates of post-traumatic stress disorder. Child Abus. Negl. 32, 139-153.

McClure, E.B., Monk, C.S., Nelson, E.E., Parrish, J.M., Adler, A., Blair, R.J.R., Fromm, S., Charney, D.S., Leibenluft, E., Ernst, M., Pine, D.S., 2007. Attention modulation of fear circuit in pediatric generalized anxiety disorder. Arch. Gen. Psychiatry 64, 97-106.

Monk, C.S., McClure, E.B., Nelson, E.E., Zarahn, E., Bilder, R.M., Leibenluft, E., Charney, D.S., Ernst, M., Pine, D.S., 2003. Adolescent immaturity in attention-related brain engagement to emotional facial expressions. NeuroImage 20, 420-428.

Monk, C.S., Nelson, E.E., McClure, E.B., Mogg, K., Bradley, B.P., Leibenluft, E., Blair, R.J.R., Chen, G., Charney, D.S., Ernst, M., Pine, D.S., 2006. Ventrolateral prefrontal cortex activation and attentional bias in response to angry faces in adolescents with generalized anxiety disorder. Am. J. Psychiatry 163, 1091-1097.

Nader, K., Kriegler, J.A., Blake, D.D., Pynoos, R.S., Newman, E., Weather, F.W., 1996. Clinician Administered PTSD Scale, Child and Adolescent Version. National Center for PTSD, White River Junction, VT.

Naim, R., Abend, R., Wald, I., Eldar, S., Levi, O., Fruchter, E., Ginat, K., Halpern, P., Sipos, M.L., Adler, A.B., Bliese, P.D., Quartana, P.J., Pine, D.S., Bar-Haim, Y., 2015. Threatrelated attention bias variability and posttraumatic stress. AJP Adv. http://dx.doi. org/10.1176/appi.ajp.2015.14121579.

Nooner, K.B., Mennes, M., Brown, S., Castellanos, F.X., Leventhal, B., Milham, M.P., Colcombe, S.J., 2014. Relationship of trauma symptoms to amygdala-based functional brain changes in adolescents: J. Trauma Stress 26 (6), 784-787.

Pagliaccio, D., Luby, J.L., Gaffrey, M.S., Belden, A.C., Botteron, K.N., Harms, M.P., Barch, D.M., 2013. Functional brain activation to emotional and non-emotional faces in healthy children: evidence for developmentally undifferentiated amygdala function during the school-age period. Cogn. Affect. Behav. Neurosci. 5, 1-19. http://dx.doi. org/10.3758/s13415-013-0167-5.

Patel, R., Spreng, R.N., Shin, L.M., Girard, T.A., 2012. Neurocircuitry models of posttraumatic stress disorder and beyond: a meta-analysis of functional neuroimaging studies. Neurosci. Biobehav. Rev. 36, 2130-2142.

Pergamin-Hight, L., Naim, R., Bakermans-Kranenburg, M.J., van IJzendoorn, M.H., BarHaim, Y., 2015. Content specificity of attention bias to threat in anxiety disorders: a meta- analysis. Clin. Psychol. Rev. 35, 10-18.

Pine, D.S., Mogg, K., Bradley, B.P., Montgomery, L.A., Monk, C.S., McClure, E., Guyer, A.E., Ernst, M., Charney, D.S., Kaufman, J., 2005. Attention bias to threat in maltreated children: implications for vulnerability to stress-related psychopathology. Am. J. Psychiatry 162, 291-296.

Price, R.B., Siegle, G.J., Silk, J.S., Ladouceur, C.D., McFarland, A., Dahl, R.E., Ryan, N.D. 2014. Looking under the hood of the dot-probe task: an fMRI study in anxious youth. Depression Anxiety 31 (3), 178-187.

Romens, S.E., Pollak, S.D., 2012. Emotion regulation predicts attention bias in maltreated children at-risk for depression. J. Child Psychol. Psychiatry 53 (2), 120-127. http:// dx.http://dx.doi.org/10.1111/j.1469-7610.2011.02474.x.

Sedlak, A.J., 2001. A History of the National Incidence Study of Child Abuse and Neglect. U.S. Department of Health and Human Services, Washington, DC.

Shin, L.M., Liberzon, I., 2010. The neurocircuitry of fear, stress and anxiety disorders. Neuropsychopharmacol. Rev. 35, 169-191.

Silverman, W.K., Saavedra, L.M., Pina, A.A., 2001. Test-retest reliability of anxiety symptoms and diagnoses with the anxiety disorders interview schedule for DSM-IV Child and Parent versions. J. Am. Acad. Child Adolesc. Psychiatry 40 (8), 937-944.

Smith, S.R., Carlson, E.B., 1996. Reliability and validity of the adolescent dissociative experiences scale. Dissociation 9 (2), 125-129.

Stoltenborgh, M., Van IJzendoorn, M.H., Euser, E.M., Bakermans-Kranenburg, M.J., 2011. Global perspective on Child Sexual Abuse: meta-analysis of prevalence around the world. Child Maltreat. 16 (2), 79-101.

Swartz, J.R., Phan, K.L., Angstadt, M., Klumpp, H., Fitzgerald, K.D., Monk, C.S., 2014a. Altered activation of the rostral anterior cingulate cortex in the context of emotional face distractors in children and adolescents with anxiety disorders. Depression Anxiety 31 (10), 870-879.

Swartz, J.R., Phan, K.L., Angstadt, M., Fitzgerald, K.D., Monk, C.S., 2014b. Dynamic changes in amygdala activation and functional connectivity in children and adolescents with anxiety disorders. Dev. Psychopathol. 26, 1305-1319.

Tahmasebi, A.M., Artiges, E., Banaschewski, T., Barker, G.J., Bruehl, R., Buchel, C., et al., 2012. Creating probabilistic maps of the face network in the adolescent brain: a multicenter functional MRI study. Hum. Brain Mapp. 33, 938-957.

Timbremont, B., Braet, C., Dreessen, L., 2004. Assessing depression in youth: relation between the Children's Depression Inventory and a structured interview. J. Clin. Child Adolesc. Psychol. 33 (1), 149-157.

Talairach, J., Tournoux, P., 1988. Co-Planar Stereotaxic Atlas of the Human Brain. 3Dimensional Proportional System: an Approach to Cerebral Imaging. Thieme, New York NY.

Telzer, E.H., Mogg, K., Bradley, B.P., Ernst, M., Pine, D.S., Monk, C.S., 2008. Relationship between trait anxiety, prefrontal cortex, and attention bias to angry faces in children and adolescents. Biol. Psychol. 79 (2), 216-222.

Van den Bulk, B.G., Koolschijn, P.C.M.P., Meens, P.H.F., Van Lang, N.D.J., Van der Wee, N.J.A., Rombouts, S.A.R.B., Vermeiren, R.R.J.M., Crone, E.A., 2013. How stable is activation in the amygdala and prefrontal cortex in adolescence? A study of emotional face processing across three measurements. Dev. Cogn. Neurosci. 4, 65-76.

Van den Bulk, B.G., Meens, P.H.F., Van Lang, N.D.J., De Voogd, E.L., Van der Wee, N.J.A., Rombouts, S.A.R.B., Crone, E.A., Vermeiren, R.R.J.M., 2014. Amygdala activation during emotional face processing in adolescents with affective disorders: the role of underlying depression and anxiety symptoms. Front. Hum. Neurosci. 8 (393), 1-13.

Van Hoof, M.J., Van Lang, N.D.J., Speekenbrink, S., Van IJzendoorn, M.H., Vermeiren, R.R.J.M., 2015. Adult Attachment Interview differentiates adolescents with Childhood Sexual Abuse from those with clinical depression and non-clinical controls. Attach. Hum. Dev. 17 (4), 354-375.

Waters, A.M., Bradley, B.P., Mogg, K., 2014. Biased attention to threat in paediatric anxiety disorders (generalized anxiety disorder, social phobia, specific phobia, separation anxiety disorder) as a function of 'distress' versus 'fear' diagnostic categorization. Psychol. Med. 44, 607-616.

Wechsler, D., 1991. The Wechsler Intelligence Scale for Children-III. Harcourt Brace, Canada.

Wechsler, D., 1997. Wechsler Adult Intelligence Scale-III. Harcourt Assessment, SanAntonio, TX.

Wolf, R.C., Herringa, R.J., 2016. Prefrontal-amygdala dysregulation to threat in pediatric posttraumatic stress disorder. Neuropsychopharmacology 41, 822-831. 